Zbigniew Grande*, Institute of Mathematics, Pedagogical University, Plac

Weyssenhoffa 11, 85-072 Bydgoszcz, Poland. e-mail:

grande@wsp. bydgoszcz.pl

\title{
ON DISCRETE LIMITS OF SEQUENCES OF FUNCTIONS SATISFYING SOME SPECIAL APPROXIMATE QUASICONTINUITY CONDITIONS
}

\begin{abstract}
In this article we investigate some properties of discrete limits of sequences of functions satisfying some special approximate quasicontinuity conditions.
\end{abstract}

Let $\mathbb{R}$ be the set of all reals. In article [2] the authors introduced the notion of the discrete convergence of sequences of functions and investigated the discrete limits in different families; for example, in the family $\mathcal{C}$ of all continuous functions.

We will say that a sequence of functions $f_{n}: \mathbb{R} \rightarrow \mathbb{R}, n=1,2, \ldots$, discretely converges to the limit $f\left(f=d-\lim _{n \rightarrow \infty} f_{n}\right)$ if

$$
\forall_{x} \exists_{n(x)} \forall_{n>n(x)} f_{n}(x)=f(x) .
$$

For any family $\mathcal{P}$ denote by $B_{d}(\mathcal{P})$ the family of all discrete limits of sequences of functions taken from the family $\mathcal{P}$.

In [2] the class $B_{d}(\mathcal{C})$ is described and the authors observe that every strictly increasing function $F$ whose set of discontinuity points is dense does not belong to the discrete Baire system generated from $\mathcal{C}$ with discrete convergence.

In this article we will investigate the discrete limits of sequences of functions satisfying some special conditions introduced in [3].

Key Words: Density topology, a.e. topology, strong quasicontinuity, discrete convergence.

Mathematical Reviews subject classification: 26A15, 26A21, 26A99.

Received by the editors July 8, 1999

*Partially supported by Bydgoszcz Pedagogical University grant 1999 
Recall that $x$ is a density point of a set $A \subset \mathbb{R}$ if there is a measurable (in the Lebesgue sense) set $B \subset A$ such that

$$
\lim _{h \rightarrow 0^{+}} \frac{\mu(B \cap(x-h, x+h))}{2 h}=1,
$$

where $\mu$ denotes Lebesgue measure in $\mathbb{R}$.

The family

$$
T_{d}=\left\{A \subset \mathbb{R} ; \forall_{x \in A} x \text { is a density point of } A\right\}
$$

is a topology called the density topology ([1] and [8]).

If $T_{e}$ denotes the Euclidean topology in $\mathbb{R}$, then the continuity of functions from $\left(\mathbb{R}, T_{d}\right)$ to $\left(\mathbb{R}, T_{e}\right)$ is called approximate continuity.

The following conditions were introduced in [3].

A function $f: \mathbb{R} \rightarrow \mathbb{R}$ satisfies the condition:

$\left(s_{1}\right)$ for every positive real $\eta$ for each point $x$ and for each set $U \in T_{d}$ including $x$ there is point $u \in U$ of continuity of $f$ such that $|f(u)-f(x)|<\eta$;

$\left(s_{2}\right)$ for every positive real $\eta$ for each point $x$ and for each set $U \in T_{d}$ including $x$ there is an open interval $I$ such that

$$
U \cap I \neq \emptyset, \quad f(I \cap U) \subset(f(x)-\eta, f(x)+\eta) \quad \text { and } \quad I \cap U \subset C(f),
$$

where $C(f)$ denotes the set of all continuity points of $f$;

$\left(s_{3}\right)$ for every positive real $\eta$ for each point $x$ and for each set $U \in T_{d}$ including $x$ there is a point $u \in U$ of approximate continuity of $f$ such that $\mid f(u)-$ $f(x) \mid<\eta$;

$\left(s_{4}\right)$ for every positive real $\eta$ for each point $x$ and for each set $U \in T_{d}$ including $x$ there is an open interval $I$ such that

$$
U \cap I \neq \emptyset, \quad f(U \cap I) \subset(f(x)-\eta, f(x)+\eta) \quad \text { and } \quad I \cap U \subset A(f),
$$

where $A(f)$ denotes the set of all points at which $f$ is approximately continuous.

In [3] it was observed that a function $f$ satisfies condition $\left(s_{1}\right)$ if and only if it is strongly quasicontinuous at each point $x$; i.e., for every positive real $\eta$ and for every set $U \in T_{d}$ including $x$ there is an open interval $I$ such that

$$
I \cap U \neq \emptyset \text { and } f(I \cap U) \subset(f(x)-\eta, f(x)+\eta),
$$


satisfies condition $\left(s_{3}\right)$ if and only if it is $T_{d}$-quasicontinuous at each point $x$; i.e., for every positive real $\eta$ and for every set $U \in T_{d}$ including $x$ there is a nonempty set $V \subset U$ belonging to $T_{d}$ such that $f(V) \subset(f(x)-\eta, f(x)+\eta)$.

The definition of strong quasicontinuity was introduced in [4], where it is also proved that every strongly quasicontinuous function $f$ is almost everywhere continuous. $T_{d}$-quasicontinuous functions were investigated in [5].

It is obvious that condition $\left(s_{2}\right)$ implies condition $\left(s_{4}\right)$ and that condition $\left(s_{4}\right)$ implies $\left(s_{3}\right)$ and $\left(s_{1}\right)$.

Remark 1. For a given nonempty set $U \in T_{d}$ and functions $f, g: \mathbb{R} \rightarrow \mathbb{R}$ we suppose that $f$ and $g$ satisfy condition $\left(s_{1}\right)$ and $U \subset A(f)$. If there is a set $V \subset \operatorname{cl}(U)$ dense in the closure $\operatorname{cl}(U)$ of the set $U$ such that $f(x)=g(x)$ for each point $x \in V$, then $f(x)=g(x)$ for each point $x \in U$.

Proof. Since the restricted functions $f \uparrow V$ and $g \uparrow V$ are equal, $f(x)=g(x)$ for each point $x \in C(f) \cap C(g) \cap U$. But $f$ and $g$ are almost everywhere continuous; so $f(x)=g(x)$ for almost all points $x \in U$. Assume, to the contrary, that there is a point $u \in U$ such that $f(u) \neq g(u)$. Let $3 \eta=$ $|f(u)-g(u)|$. There is a closed set

$$
A \subset U \cap\{x ;|f(u)-f(x)|<\eta\} \cap\{x ; f(x)=g(x)\}
$$

such that $u$ is a density point of $A$. Since $g$ satisfies condition $\left(s_{1}\right)$, the point $u$ is not any density point of the interior $\operatorname{int}(\{x ;|g(x)-g(u)| \geq \eta\})$ of the set $\{x ;|g(x)-g(u)| \geq \eta\}$. So $u$ is a density point of the set $A \cap\{x ;|f(x)-f(u)|<\eta\}$ and $u$ is not a density point of the set $\{x ;|g(x)-g(u)| \geq \eta\}$. Thus

$$
A \cap\{x ;|f(x)-f(u)|<\eta\} \cap\{x ;|g(x)-g(u)|<\eta\} \neq \emptyset
$$

and there is a point $w \in A$ such that $|f(w)-f(u)|<\eta$ and $|g(w)-g(u)|<\eta$. Since $f(w)=g(w)$, this implies

$$
3 \eta=|f(u)-g(u)| \leq|f(u)-f(w)|+|g(w)-g(u)|<2 \eta,
$$

which is a contradiction and thus completes the proof.

Theorem 1. If a function $f: \mathbb{R} \rightarrow \mathbb{R}$ is the discrete limit of a sequence of functions $f_{n}, n=1,2, \ldots$, satisfying condition $\left(s_{1}\right)$, then it satisfies the following condition

$\left(i_{1}\right)$ for each nonempty set $A \in T_{d}$ there is an open interval $I$ such that $I \cap A \neq \emptyset$, the restricted function $f \uparrow(I \cap A)$ is almost everywhere continuous and for each positive real $\eta$ and every point $x \in I \cap A$ there is an open interval $J \subset I \cap(x-\eta, x+\eta)$ for which

$$
J \cap A \neq \emptyset \text { and } f(J \cap A) \subset(f(x)-\eta, f(x)+\eta) .
$$


Proof. Let $A \in T_{d}$ be a nonempty set. For $n=1,2, \ldots$ let

$$
A_{n}=\left\{x \in \operatorname{cl}(A) ; f_{k}(x)=f(x) \text { for } k \geq n\right\} .
$$

Observe that $\operatorname{cl}(A)=\bigcup_{n=1}^{\infty} A_{n}$ and $A_{n} \subset A_{n+1}$ for $n \geq 1$. There is a positive integer $m$ such that the set $A_{m}$ is of the second category in $\operatorname{cl}(A)$. So there is an open interval $I$ with $I \cap A_{m} \neq \emptyset$ and $I \cap \operatorname{cl}(A) \subset \operatorname{cl}\left(I \cap A_{m}\right)$. Put $B=\bigcap_{k \geq m} C\left(f_{k}\right)$ and observe that for $k \geq m$ and $x \in I \cap B \cap \operatorname{cl}(A)$ we have $f_{k}(x)=f(x)$. Since the functions $f_{n}, n \geq 1$, are almost everywhere continuous, the set $(I \cap A) \backslash B$ is of measure zero. But

$$
f \uparrow(A \cap I \cap B)=f_{k} \uparrow(A \cap I \cap B) \text { for } k \geq m,
$$

so the restricted function $f \uparrow(I \cap A)$ is almost everywhere continuous.

Assume, to the contrary, that there is a positive real $\eta$ and a point $x \in I \cap A$ such that for every open interval $J \subset I \cap(x-\eta, x+\eta)$ such that $J \cap A \neq \emptyset$ there is a point $u \in J \cap A$ at which $|f(u)-f(x)| \geq \eta$. Let $j \geq m$ be an integer such that $f_{k}(x)=f(x)$ for $k \geq j$. Since $x \in(x-\eta, x+\eta) \cap I \cap A \in T_{d}$ and the function $f_{j}$ satisfies condition $\left(s_{1}\right)$, there is an open interval $J \subset I \cap(x-\eta, x+\eta)$ such that

$$
J \cap A \neq \emptyset \text { and } f_{j}(J \cap A) \subset\left(f_{j}(x)-\frac{\eta}{2}, f_{j}(x)+\frac{\eta}{2}\right) .
$$

Let $u \in J \cap A$ be a point for which $|f(u)-f(x)| \geq \eta$ and let $i \geq j$ be an integer such that $f_{k}(u)=f(u)$ for $k \geq i$. Since the function $f_{i}$ satisfies condition $\left(s_{1}\right)$ and since $u \in J \cap A \in T_{d}$, there is an open interval $K \subset J$ such that

$$
K \cap A \neq \emptyset \text { and } f_{i}(K \cap A) \subset\left(f(u)-\frac{\eta}{2}, f(u)+\frac{\eta}{2}\right) .
$$

But the restricted functions $f_{i}\lceil(J \cap A)$ and $f(J \cap A)$ are almost everywhere equal; so there is a point $w \in J \cap A \cap B$ at which the equality $f(w)=f_{i}(w)=$ $f_{j}(w)$ holds. Consequently,

$$
\eta \leq|f(u)-f(x)| \leq\left|f_{i}(u)-f_{i}(w)\right|+\left|f_{j}(w)-f_{j}(x)\right|<\frac{\eta}{2}+\frac{\eta}{2}=\eta
$$

and the contradiction completes the proof.

Since the functions satisfying condition $\left(s_{1}\right)$ are almost everywhere continuous, for the function $f$ from the last theorem there is an $F_{\sigma}$-set $E$ of measure zero such that the restricted function $f \backslash(\mathbb{R} \backslash E)$ is the discrete limit of a sequence of continuous functions on $\mathbb{R} \backslash E$ ([6]).

The next result follows from the proof of the last theorem. 
Corollary 1. If the function $f: \mathbb{R} \rightarrow \mathbb{R}$ is the discrete limit of a sequence of functions $f_{n}, n \geq 1$, satisfying condition $\left(s_{1}\right)$, then the set

$$
D_{s q}(f)=\{x ; f \text { is not strongly quasicontinuous at } x\}
$$

is nowhere dense.

Proof. Let $I$ be an open interval. As in the proof of the last theorem, we find an open interval $J \subset I$ a set $E \subset J$ of measure zero and a positive integer $m$ such that $f_{k}(x)=f(x)$ for $x \in J \backslash E$ and $k \geq m$. The reasoning used in the proof of the last theorem shows that $f$ is strongly quasicontinuous at each point $x \in J$.

Theorem 2. If a function $f: \mathbb{R} \rightarrow \mathbb{R}$ is the discrete limit of a sequence of functions $f_{n}, n \geq 1$, satisfying condition $\left(s_{4}\right)$, then it satisfies the following condition.

$\left(i_{2}\right)$ For every nonempty set $A \in T_{d}$ there are an open interval $I$ and a positive integer $m$ such that

$$
A(f) \supset I \cap A \neq \emptyset \text { and } f_{k}(x)=f(x) \text { for } ; x \in A \cap I \text { and } k \geq m \text {. }
$$

Proof. As in the proof of Theorem 1, we find a positive integer $m$ and an open interval $I$ such that $\emptyset \neq I \cap A \subset A\left(f_{m}\right)$ and $\bigcap_{k \geq m}\left\{x \in I \cap A ; f_{k}(x)=\right.$ $f(x)\}$ is dense in $I \cap A$. Now we use Remark 1 and observe that $f_{k}(x)=f(x)$ for $x \in I \cap A$ and $k \geq m$.

As an immediate consequence we obtain the following corollary.

Corollary 2. For the function $f$ from Theorem 2 the set $D_{s q}(f) \cap(\mathbb{R} \backslash A(f))$ is nowhere dense.

Theorem 3. If a function $f: \mathbb{R} \rightarrow \mathbb{R}$ is the discrete limit of a sequence of functions $f_{n}, n=1,2, \ldots$, satisfying condition $\left(s_{2}\right)$, then it satisfies the following condition.

$\left(i_{3}\right)$ For each nonempty set $A \in T_{d}$ there is an open interval $I$ such that $I \cap A \neq \emptyset$ and the restricted function $f \uparrow(I \cap A)$ is continuous.

Proof. The proof is completely analogous to that of Theorem 2.

As an immediate consequence we obtain the following.

Corollary 3. If a function $f: \mathbb{R} \rightarrow \mathbb{R}$ is the discrete limit of a sequence of functions $f_{n}, n \geq 1$, satisfying condition $\left(s_{2}\right)$, then the set $\mathbb{R} \backslash C(f)$ is nowhere dense. 
Let $f: \mathbb{R} \rightarrow \mathbb{R}$ be an increasing function whose set of discontinuities is dense. If for all discontinuities $x$ of $f$ we suppose that

$$
f(x)=\frac{\lim _{t \rightarrow x^{+}} f(t)+\lim _{t \rightarrow x^{-}} f(t)}{2},
$$

then $f$ is not in the Baire system generated by the family of all functions satisfying condition $\left(s_{1}\right)$ and discrete convergence.

Of course, for every quasicontinuous function $g$ the set $\{x ; f(x) \neq g(x)\}$ is residual, so the graph of the function $f$ can not covered by a countable family of the graphs of quasicontinuous functions, and consequently $f$ does not belong to the above Baire system.

However if for all discontinuity points $x$ of $f$ we have $f(x)=\lim _{t \rightarrow x^{+}} f(t)$, then $f$ satisfies condition $\left(s_{1}\right)$, but is not the discrete limit of any sequence of functions satisfying condition $\left(s_{4}\right)$, because the set $\mathbb{R} \backslash A(f)$ is dense.

Every almost everywhere continuous function $h: \mathbb{R} \rightarrow \mathbb{R}$ which is everywhere approximately continuous and discontinuous on a dense set, satisfies condition $\left(s_{4}\right)$, but is not the discrete limit of any sequence of functions satisfying condition $\left(s_{2}\right)$, because its set of discontinuities is dense.

Theorem 4. A function $f: \mathbb{R} \rightarrow \mathbb{R}$ is the discrete limit of a sequence of functions satisfying condition $\left(s_{3}\right)$ if and only if it is measurable.

Proof. The necessity is evident, since all functions satisfying condition $\left(s_{3}\right)$ are measurable. The proof of the sufficiency is the repetition of the proof for the pointwise limits from [7] or [5].

If the function $f$ is measurable, then the set $B=\mathbb{R} \backslash A(f)$ is of measure zero. Let $E \supset B$ be a $G_{\delta}$-set of measure zero. There are ([7]) measurable sets $B_{n, k}, k, n=1,2, \ldots$, such that

$$
B_{n, k} \cap B_{m, l}=\emptyset \text { if }(n, k) \neq(m, l) \text { and } \mathbb{R} \backslash E=\bigcup_{k, n=1}^{\infty} B_{n, k} ;
$$

if $x \in B_{n, k} \cup E$, then $x$ is not a density point of the set $\mathbb{R} \backslash B_{n, k} \backslash E$, $k, n=1,2, \ldots$.

Let $\left(w_{n}\right)$ be an enumeration of all rationals such that $w_{n} \neq w_{m}$ for $n \neq m$. For $n \geq 1$ define $f_{n}$ by

$$
f_{n}(x)= \begin{cases}w_{k} & \text { if } x \in B_{n, k} \text { for } k=1,2, \ldots \\ f(x) & \text { otherwise on } \mathbb{R} .\end{cases}
$$

Then each function $f_{n}, n \geq 1$, satisfies condition $\left(s_{3}\right)$ and the sequence $\left(f_{n}\right)$ discretely converges to $f$. 
If $C$ is a Cantor set of positive measure such that for each open interval $I$ with $I \cap C \neq \emptyset$ the set $I \cap C$ is of positive measure and if $B \subset C$ is a countable set such that every point $x \in B$ is a density point of $C$ and $\operatorname{cl}(B)=C$, then the function $f$ equal 1 on $B$ and zero otherwise on $\mathbb{R}$ does not satisfy condition $\left(i_{1}\right)$. So, it is not the discrete limit of any sequence of functions satisfying condition $\left(s_{1}\right)$.

Evidently $f$ is the discrete limit of a sequence of almost everywhere continuous functions.

Theorem 5. Let $f: \mathbb{R} \rightarrow \mathbb{R}$ be a function. Suppose that there are pairwise disjoint closed sets $A_{n}$ of measure zero and functions $g_{n}: \mathbb{R} \rightarrow \mathbb{R}, n=1,2, \ldots$, satisfying condition $\left(s_{j}\right)$, where $j \in\{1,2,4\}$, on the sets $\mathbb{R} \backslash A_{n}$, such that the restricted function $f \uparrow\left(\mathbb{R} \backslash \bigcup_{n=1}^{\infty} A_{n}\right)$ is the discrete limit of the sequence of restricted functions $g_{n} \uparrow\left(\mathbb{R} \backslash \bigcup_{k=1}^{\infty} A_{k}\right)$ and is not a point $x \in \bigcup_{k=1}^{\infty} A_{k}$ being a density point of the closure $\operatorname{cl}\left(\bigcup_{k=1}^{\infty} A_{k}\right)$. Then $f$ is the discrete limit of a sequence of functions satisfying condition $\left(s_{j}\right)$.

Proof. Evidently the set $A=\bigcup_{n=1}^{\infty} A_{n}$, is nowhere dense. We find pairwise disjoint closed intervals $I_{n, k, m}=\left[a_{n, k, m}, b_{n, k, m}\right], k, n, m=1,2, \ldots$, such that:

$I_{n, k, m} \cap \operatorname{cl}(A)=\emptyset$ for $m, n, k \geq 1$;

all endpoints $a_{n, k, m}, b_{n, k, m}$ are continuity points of $g_{n}, k, m, n=1,2, \ldots$;

if $x$ is an accumulation point of the set $\left\{a_{n, k, m}, b_{n, k, m} ; k, m=1,2 \ldots\right\}$, then $x \in A_{n}, n \geq 1$;

if $x \in A_{n}$ an $\left(m_{i}\right)_{i}$ is a strictly increasing sequence of positive integers, then $x$ is not a density point of the set $\mathbb{R} \backslash \bigcup_{i=1}^{\infty} I_{n, k, m_{i}}$ for $k, n \geq 1$.

In the interior of each interval $I_{k, n, m}, k, m, n \geq 1$, we find a closed interval $J_{n, k, m}$ such that for every point $x \in A_{n}$ and for every strictly increasing sequence of positive integers $m_{i}, i=1,2, \ldots, x$ is not a density point of a set $\mathbb{R} \backslash \bigcup_{i=1}^{\infty} J_{n, k, m_{i}}$ for $k, n \geq 1$. For a nonempty set $X \subset \mathbb{R}$ and for $x \in \mathbb{R}$ let

$$
\operatorname{dist}(x, X)=\inf \{|t-x| ; t \in X\}
$$

Let $\left(w_{k}\right)_{k}$ be an enumeration of all rationals. We will define functions $f_{n}$, $n=1,2, \ldots$, as follows. Fix a positive integer $n$. If $x \in J_{i, k, m}, i \leq n$, $k, m=1,2, \ldots$, and if

$$
\max \left(\operatorname { d i s t } \left(a_{i, k, m}, \bigcup_{i \leq n} A_{i}, \operatorname{dist}\left(b_{i, k, m}, \bigcup_{i \leq n} A_{i}\right)<\frac{1}{n}\right.\right.
$$


then $f_{n}(x)=w_{k}$, for $k, m, n \geq 1$. If $\operatorname{dist}\left(x, \bigcup_{i<n} A_{i}\right) \geq \frac{1}{n}$ or if $x \in \mathbb{R} \backslash$ $\bigcup_{i<n} \bigcup_{k, m>1} \operatorname{int}\left(I_{i, k, m}\right)$ or if $x \in I_{n, k, m}$ and condition $(*)$ does not hold, then $f_{n}(x)=g_{n}(x)$. If $i \leq n$ and the triple $(i, k, m)$ satisfies condition $(*)$, then $f_{n}$ is linear on the components of the set $I_{i, k, m} \backslash \operatorname{int}\left(J_{i, k, m}\right)$. Then the functions $f_{n}, n \geq 1$, satisfy condition $\left(s_{j}\right)$ and the sequence $\left(f_{n}\right)$ discretely converges to $f$.

\section{References}

[1] A. M. Bruckner, Differentiation of real functions, Lectures Notes in Math. 659, Springer-Verlag, Berlin, 1978.

[2] A. Csásár and M. Laczkovich, Discrete and equal convergence, Studia Sci. Math. Hung. 10 (1975), 463-472.

[3] Z. Grande, On some special notions of approximate quasi-continuity, Real Analysis Exchange. 24 (1998-99), 171-183.

[4] Z. Grande, Measurability, quasicontinuity and cliquishness of functions of two variables, Real Anal. Exch. 20 (1994-95), 744- 752.

[5] Z. Grande, Sur la quasi-continuité et la quasi-continuité approximative, Fund. Math. 129 (1988), 167-172.

[6] Z. Grande, On discrete limits of sequences of approximately continuous and $T_{a e}$-continuous functions, to appear.

[7] Z. Grande, T. Natkaniec and E. Strońska, Algebraic structures generated by d-quasi continuous functions, Bull. Pol. Acad. Sci., Math. 35 (1987), $717-723$.

[8] F. Tall, The density topology, Pacific J. Math. 62 (1976), 275-283. 